\title{
Troisième édition du CoBioMe Congrès des internes de Biologie médicale Paris, 7 mars 2020
}

\author{
Charles Marchionini \\ Amandine Caillault \\ Théophile Cocherie \\ Floriane Devaux \\ Lise Larcher \\ Edouard Le Guillou \\ Lorra Monpierre \\ Céline Mory \\ Clémentine Moulin \\ Laurence Pacot \\ Inès Rezzoug \\ Audrey Sabbagh \\ Bérénice Schell \\ Marie Temple \\ Pauline Thiebot \\ Hélène Vantomme \\ Eve-Marie Walle \\ Syndicat des internes en pharmacie \\ et biologie médicale d'lle-de-France, \\ Paris, France
}

\section{Le mot du comité d'organisation}

Charles Marchionini, Pauline Thiebot, Hélène Vantomme

Pour la troisième année consécutive, le Syndicat des internes en pharmacie et biologie médicale d'île-de-France (SIPHIF) a organisé son propre congrès CoBioMe le samedi 7 mars 2020 à l'hôpital Saint-Louis à Paris en partenariat avec les Annales de Biologie Clinique.

Comme chaque année, les internes de biologie médicale d'Ile-de-France ont pu soumettre leur résumé dans l'une des trois catégories que sont «Biologie hospitalière ", « Recherche fondamentale, translationnelle et clinique », et « Cas cliniques originaux ». À la suite de ces soumissions, le comité scientifique a pu sélectionner 3 résumés pour

Correspondance :

<congrèsbiosiphif@gmail.com> la réalisation d'une communication longue de 10 minutes maximum dans chacune des catégories et 6 résumés pour une communication flash de 3 minutes.

À l'issue de cette journée, le comité scientifique du congrès a pu délibérer et attribuer un prix par catégorie pour les communications longues et un prix pour une communication flash.

Par ailleurs, l'interne ayant remporté le prix dans la catégorie « cas clinique original » en publication longue aura son travail publié sous forme d'un article original dans un prochain numéro des Annales de Biologie Clinique.

Le CoBioMe est devenu un évènement privilégié pour les internes de biologie médicale afin de valoriser leurs travaux, communiquer dans un contexte scientifique et récompenser l'implication des internes dans des travaux académiques. Cette journée est à présent un incontournable de la vie de l'internat de biologie médicale à Paris. 


\section{Session « Cas cliniques originaux »}

\section{Prolifération de cellules dendritiques plasmocytoédes associée... une hémopathie : autour d'un cas}

Marie Temple ${ }^{1}$, Jennifer Osman ${ }^{1}$, Rémy Favre ${ }^{1}$, Sophie Rigaudeau $^{2}$, Francine Garnache-Ottou ${ }^{3}$, Victoria Raggueneau ${ }^{1}$, Benjamin Maneglier ${ }^{l}$

Service de biochimie générale, CHU de Rouen, France ; 1 Service de biologie médicale, Centre hospitalier André Mignot, Le Chesnay, France ; 2 Service d'hématologie, Centre hospitalier André Mignot, Le Chesnay, France ; 3 Laboratoire de cytologie, Etablissement français du sang, Besançon, France

Les cellules dendritiques plasmacytoïdes (pDC) sont des cellules immunomodulatrices sécrétant de l'interféron alpha et interagissant avec les lymphocytes T. La classification OMS 2017 distingue deux entités pathologiques : les proliférations de précurseurs de $\mathrm{pDC}$, à l'origine de la leucémie à $\mathrm{pDC}$ et les proliférations de $\mathrm{pDC}$ matures (MPDCP) associées à une hémopathie myéloïde (entité provisoire).

Monsieur M., 57 ans, en rémission complète d'un myélome multiple stade III, est actuellement en traitement d'entretien par daratumumab. Il consulte pour la dernière cure de son traitement. À l'examen clinique, le patient présente des pétéchies, sans syndrome tumoral ni autre lésion cutanée, motivant la réalisation d'une numération sanguine (NFS). La NFS retrouve une anémie macrocytaire $(7,4 \mathrm{~g} / \mathrm{dL}$ VU : $13-18 \mathrm{~g} / \mathrm{dL})$, une thrombopénie sévère $(20 \mathrm{G} / \mathrm{L} \mathrm{VU}: 150$ $400 \mathrm{G} / \mathrm{L})$ associées à une monocytose $(3,7 \mathrm{G} / \mathrm{L}$ VU : 0,15-1 $\mathrm{G} / \mathrm{L})$ et $5 \%$ de blastes indifférenciés.

Le myélogramme montre une moelle très riche, avec une hyperplasie de la lignée granuleuse (neutrophiles, éosinophiles et monocytes) et des signes de dysgranulopoïèse, sans excès de plasmocytes. On observe également $13 \%$ de blastes lymphoïdes B de phénotype CD19+22+/10-, exprimant de manière aberrante un marqueur myéloïde (CD13+), ainsi qu'un contingent de cellules atypiques représentant $8 \%$ des cellules médullaires, co-exprimant les antigènes CD4+123+303+304+FcER1+BadLamp+CD43+CD7+

sans expression de l'antigène cTCL1. Leur morphologie et immunophénotype (CD56-) correspondent à des pDC matures. Les analyses cytogénétiques et moléculaires mettent en évidence un remaniement de KMT2A (MLL) avec ARHGEF12, translocation décrite une seule fois dans un cas de leucémie aiguë myéloïde (LAM), ainsi que des mutations habituellement décrites dans les hémopathies myéloïdes (NRASG13D, DNMT3AN757D et R792 et RUNX1F136C). Le transcrit de fusion BCR-ABL n'a pas été retrouvé. Le TEP scan est en faveur d'une hémopathie exclusivement médullaire.

Les MPDCP associées à une hémopathie sont très rares (80 cas publiés), de mauvais pronostic, plus fréquemment diagnostiquées chez l'homme avec un âge médian au diagnostic de 69 ans. L'hémopathie myéloïde la plus souvent associée est la leucémie myélomonocytaire chronique (LMMC), mais des associations avec des syndromes myélodysplasiques ou des LAM ont aussi été rapportées.

Nous décrivons ici un cas de MPDCP associée à une probable LMMC et à un excès de lymphoblastes $\mathrm{B}$, chez un patient traité et autogreffé pour un myélome multiple. Un autre cas de MPDCP associé à une LMMC et une leucémie aiguë lymphoblastique $\mathrm{T}$ a été documenté dans la cohorte française. Les données de cette cohorte (en cours d'analyse) montrent, sur des cas similaires, des profils mutationnels communs entre les différentes populations blastiques, pDC et monocytaires. Après concertation pluridisciplinaire, un traitement par tagraxofusp (anti-CD123) suivie d'une allogreffe de cellules souches hématopoïétiques a été décidé.

\section{Pandoraea pnomenusa : un pathogène méconnu dans la mucoviscidose? À propos d'un cas}

Théophile Cocherie, Pauline Bargain, Emmanuelle Coirier-Duet, Anne-Gaëlle Cauchie, Amandine Henry, Stéphanie Marque-Juillet, Marlène Amara

Service de microbiologie, $\mathrm{CH}$ André Mignot de Versailles, Le Chesnay, France

\section{L'observation}

Un enfant de 9 ans atteint de mucoviscidose présente une altération de l'état général avec toux persistante, perte de poids et majoration d'une dyspnée d'effort. Il est hospitalisé pour éradication d'une colonisation pulmonaire à Pseudomonas aeruginosa objectivée à Dubaï, son lieu de résidence. Un examen cytobactériologique des crachats (ECBC) effectué sous ciprofloxacine est réalisé au début de son hospitalisation. La mise en culture de ce prélèvement permet d'isoler des germes pour lesquels le patient était connu colonisé : Staphylococcus aureus et Pseudomonas aeruginosa. Le milieu cepacia (Oxoid) permet de mettre en évidence une primo colonisation à Burkholderia cepacia à 10,2 UFC/mL et à Pandoraea pnomenusa à 10,5 UFC/mL. Toutes les identifications ont été réalisées par spectrométrie de masse Microflex (Bruker). Le patient présente une amélioration clinique sous traitement avec une diminution de la toux associée à une prise de poids. Cependant, les ECBC de contrôle à $\mathrm{J} 2$ puis $\mathrm{J} 6$ demeurent positifs à P. pnomenusa et à $S$. aureus. La souche isolée présente une 
sensibilité à l'imipénem, la lévofloxacine, au cotrimoxazole et à la minocycline et une résistance à la témocilline, ceftazidime, céfépime, ceftolozane-tazobactam, tigécycline et ciprofloxacine. En l'absence de recommandations spécifiques à Pandoraea, les diamètres d'inhibition et les CMI ont été interprétés selon les recommandations en vigueur pour Burkholderia lorsqu'elles existaient ou selon les concentrations critiques PK-PD du CASFM 2019 v2.0. Une quadrithérapie par ceftazidime, tobramycine, lévofloxacine et cotrimoxazole a été introduite à $\mathrm{J} 8$ afin de maintenir une bithérapie sur chaque type de germe, permettant une négativation complète des prélèvements à partir de J19.

\section{Discussion}

Décrit en 2000, Pandoraea est un bacille à Gram négatif non fermentant de l'environnement. Il est proche des Burkholderia et Ralstonia, connus pour leurs rôles dans les exacerbations pulmonaires et le déclin des fonctions respiratoires chez les patients atteints de mucoviscidose. L'isolement de Pandoraea est beaucoup plus rare et son identification précise a été améliorée depuis l'utilisation de la spectrométrie de masse en microbiologie médicale. L'Observatoire national des Burkholderia recense en moyenne 5 cas par an d'infection à Pandoraea, principalement $P$. sputorum et à notre connaissance il s'agit du premier cas français d'isolement de $P$. pnomenusa chez un patient atteint de mucoviscidose. Seuls deux cas ont été décrits dans la littérature et l'isolement de ce germe semblait associé à un pronostic péjoratif. Cependant, le rôle pathogène de Pandoraea reste méconnu compte tenu du faible nombre de cas. Les praticiens impliqués dans le diagnostic et le traitement des patients atteints de mucoviscidose doivent connaître son importance clinique, en effet, comme Burkholderia, une colonisation chronique à Pandoraea correspond à un tournant majeur dans l'évolution des patients atteints de mucoviscidose.

\section{Difficulté dans l'interprétation d'une intoxication au cyanure, toujours d'actualité : ... à propos d'un cas}

Céline Mory ${ }^{l}$, Adeline Knapp ${ }^{1}$, Charlotte MayerDuverneuil $^{1}$, Jean Claude Alvarez ${ }^{1,2}$

1 Laboratoire de toxicologie, Hôpital Raymond Poincaré, Garches, France ; 2 Plateforme MasSpecLab, UMR1173, Inserm, Montigny-le-Bretonneux, France

La contribution de la toxicologie analytique dans le diagnostic d'intoxication au cyanure $(\mathrm{CN})$ est perturbée par l'interprétation délicate voire infructueuse des concentrations sanguines chez le patient vivant ou en post-mortem, notamment après administration d'antidote. Nous rapportons ici un cas de décès après prise en charge médicale hospitalière par ingestion de sels de $\mathrm{CN}$ documenté par des prélèvements sanguins ante-mortem et post-mortem. Un homme de 34 ans est découvert en arrêt cardio-respiratoire avec vomissements abondants et sanguinolents après ingestion supposée d'un flacon de $100 \mathrm{~mL}$ de réactif alcalin à base de $\mathrm{NaCN}$ utilisé pour le titrage du manganèse. Le flacon retrouvé sur les lieux a incité le Samu à administrer rapidement une dose de $5 \mathrm{~g}$ d'hydroxocobalamine (Cyanokit@), l'antidote de l'intoxication au $\mathrm{CN}$, permettant de récupérer rapidement une activité cardiaque spontanée. À l'arrivée aux urgences, on observe un érythème cutané généralisé associé au liquide lacrymal rouge vif dû à l'injection de Cyanokit ${ }^{\circledR}$, une alcoolémie à $2,1 \mathrm{~g} / \mathrm{L}$ avec reste du bilan toxicologique négatif. Le bilan biologique révèle une acidose lactique sévère (lactates à $16 \mathrm{mM}$ ) et certaines analyses sont perturbées par la coloration rouge vif de l'hydroxocobalamine. L'évolution est rapidement défavorable conduisant au décès $7 \mathrm{~h}$ après son admission, malgré une seconde dose de Cyanokit ${ }^{\text {. Le rap- }}$ port d'autopsie révèle une mort toxique par ingestion de produits à l'origine d'une altération de la muqueuse gastroœsophagienne témoignant de lésions caustiques typiques des produits alcalins associé à un syndrome asphyxique et une coloration anormale des viscères. L'analyse du produit montre une concentration de $35 \mathrm{~g} / \mathrm{L}$ de $\mathrm{CN}$, correspondant à une dose supposée ingérée de 3,5 $\mathrm{g}$ (dose létale : 0,1 à $0,2 \mathrm{~g}$ ). Les prélèvements suivants sont analysés par LC-MS/MS après dérivation du CN : 1 échantillon antemortem (1) $4 \mathrm{~h}$ après intoxication et $2 \mathrm{~h}$ après injection de Cyanokit ${ }^{\circledR}$ et 2 échantillons post-mortem (2) 10 min après le décès et $8 \mathrm{~h}$ après injection de Cyanokit ${ }^{\circledR}$; (3) lors de l'autopsie, $6 \mathrm{j}$ après le décès. Les concentrations en $\mathrm{CN}$ (CCN) sont : (1) $\mathrm{CCN}=5,8 \mu \mathrm{g} / \mathrm{mL}$; (2) $\mathrm{CCN}=0,26$ $\mu \mathrm{g} / \mathrm{mL}$; (3) $\mathrm{CCN}=0,075 \mu \mathrm{g} / \mathrm{mL}$. L'interprétation diffère selon les prélèvements : (1) CCN considérée comme létale (2) $\mathrm{CCN}$ légèrement supérieure aux $\mathrm{CCN}$ physiologiques (3) CCN considérée comme physiologique $(<0,2 \mu \mathrm{g} / \mathrm{mL})$ pouvant écarter à tort une intoxication au $\mathrm{CN}$ en l'absence de contexte. L'hydroxocobalamine est responsable d'une diminution de la $\mathrm{CCN}$ après formation de cyanocobalamine, complexe non toxique éliminé par le rein. Cette diminution semble se poursuivre en post-mortem. Cependant, le $\mathrm{CN}$ a provoqué des dommages mitochondriaux cellulaires irréversibles alors qu'il a totalement disparu du sang. L'administration d'antidote, les problèmes de stabilité et les difficultés analytiques rendent délicate l'interprétation des résultats et doivent être pris en compte lors de suspicions d'intoxication au $\mathrm{CN}$. 


\section{Efficacité d'un traitement par anticoagulant oral direct et immunoglobulines intraveineuses dans un cas complexe de thrombopénie induite ... à l'héparine immuno-allergique résistante aux traitements usuels}

Eve-Marie Walle ${ }^{1}$, Olivier Chassin ${ }^{2}$, Nicolas Legris ${ }^{2}$, Noémie Chanson ${ }^{3}$, Bernard Jondeau ${ }^{1}$, Andreas PerrierCornet $^{l}$, Cécile Lavenu-Bombled ${ }^{l}$, Sophie Combe ${ }^{1}$, Véronique Picard ${ }^{1}$, Safa Sanekli ${ }^{1}$, Valérie Proulle ${ }^{1}$

1 Service d'hématologie biologique, 2 Service de neurologie adultes, 3 Service de médecine interne, CHU Bicêtre, HUPS, AP-HP, Université Paris-Saclay, Le KremlinBicêtre, France

Nous présentons le cas d'une thrombopénie induite à l'héparine immuno-allergique (TIH) dont la prise en charge a nécessité le relais du danaparoïde sodique (DS) par un anticoagulant oral direct (AOD) et l'administration d'immunoglobulines intraveineuses (IGIV). Le patient est un homme de 71 ans polyvasculaire (HTA, diabète, coronaropathie, artériopathie). Il est traité par antibiotiques pour un érysipèle du membre inferieur (MI), mais est hospitalisé 8 jours après pour aggravation clinique. Une thrombose veineuse profonde des MI (TVP) est éliminée et une anticoagulation préventive par héparine (enoxaparine sodique, HBPM) débutée. A J7 du traitement par HBPM, le patient présente une hémiplégie gauche, reliée à une ischémie cérébrale carotidienne objectivée sur l'IRM, et une chute brutale des plaquettes (74 à $20 \mathrm{G} / \mathrm{L}$ ). L'association d'une diminution de plus de $50 \%$ du chiffre de plaquettes et d'une thrombose aiguë à $\mathrm{J} 7$ d'un traitement par HBPM suggère une TIH (score de $4 \mathrm{~T}=6$ ) confirmée par la détection d'anticorps anti-PF4 par test Elisa. Le traitement par HBPM est arrêté et relayé par le DS (recommandations GIHP/GFHT 2019), mais à doses préventives malgré la thrombose aiguë, associé à des transfusions plaquettaires et une supplémentation en fibrinogène. Il est alors transféré dans notre hôpital. A l'arrivée, il présente une thrombopénie $(49 \mathrm{G} / \mathrm{L})$, un TP à $59 \%$, un fibrinogène à $1,4 \mathrm{~g} / \mathrm{L}$ et des D-dimères > $4000 \mathrm{ng} / \mathrm{mL}$. Le DS est administré à doses curatives. Mais le tableau thrombotique s'aggrave avec apparition de nouvelles lésions ischémiques cérébrales à l'IRM. A J12, devant l'extension de la thrombose cérébrale et la persistance de la thrombopénie associée à des D-dimères élevés, une TIH par réaction croisée au DS et une cause autre de CIVD (septique) sont évoquées. Le DS est relayé par un AOD, le dabigatran, comme suggéré dans la littérature (Theodore, 2017). Le diagnostic de TIH croisée est éliminé par la négativité du test spécifique de relargage à la sérotonine marquée. A l'inverse, un sepsis sur angiocholite aiguë est diagnostiqué et l'antibiothérapie probabiliste instaurée est efficace en $48 \mathrm{~h}$. Le traitement par AOD est maintenu, mais la thrombopénie persiste (16 G/L). À J5 du traitement curatif par AOD, malgré des transfusions plaquettaires, survient un épisode de rectorragie nécessitant une transfusion érythrocytaire. Un traitement par IGIV est débuté, comme proposé dans la littérature (Greinacher, 2017) pour certaines TIH auto-immunes, qui permet une ascension rapide du chiffre de plaquettes en $48 \mathrm{~h}$ ( 66 puis $163 \mathrm{G} / \mathrm{L}$ ). En raison d'une TVP du MI découverte à l'échographie doppler, le traitement par AOD est maintenu sans complication pendant 3 mois.

En conclusion, notre patient a présenté une thrombopénie due à une TIH associée à une CIVD et un sepsis. La correction de la thrombopénie a nécessité l'utilisation d'IGIV. Les AOD ont été utilisés avec succès pour l'anticoagulation soulignant leur utilité dans la prise en charge de certaines TIH.

\section{Une protozoose américaine mal connue}

Lorra Monpierre $^{l^{*}}$, Olivier Voisin ${ }^{2}$, Gabriel Macheda ${ }^{3}$, Alban Le Monnier ${ }^{4}$, Benoît Pilmis ${ }^{3}$, Assaf Mizrahi ${ }^{4}$, Yaye Senghor ${ }^{1}$

\section{*Lauréat du Prix SFBC}

1 Service de microbiologie clinique, 2 Service de médecine interne, 3 Équipes mobiles de microbiologie clinique, 4 Service de microbiologie clinique, Groupe hospitalier Paris-Saint-Joseph, Paris, France ; EA4043 Unité bactéries pathogènes et santé, Université Paris-Sud Saclay, ChâtenayMalabry, France

La babésiose est une zoonose cosmopolite émergente causée par un protozoaire intra-érythrocytaire du genre Babesia dont la transmission est principalement médiée par des tiques. Plusieurs espèces de Babesia sont responsables de la maladie chez l'homme, les principales étant Babesia microti en Amérique du Nord, B. divergens et B. venatorum en Europe. La présentation clinique de la maladie varie d'une infection asymptomatique à un tableau d'infection sévère pouvant engager le pronostic vital.

Nous rapportons ici le cas d'un patient français, vivant dans le Nord Est des Etats-Unis depuis 19 ans. Il consulte dans le service d'accueil des urgences en juin 2019 pour fièvre à $38,4{ }^{\circ} \mathrm{C}$, asthénie, anorexie évoluant depuis 5 jours. À son arrivée, le bilan biologique objectivait une bicytopénie avec une anémie normocytaire à $8,6 \mathrm{~g} / \mathrm{dL}$ associée à une thrombopénie à $34000 / \mathrm{mm} 3$. Le bilan sanguin révélait également un syndrome inflammatoire biologique avec une CRP à $270 \mathrm{mg} / \mathrm{L}$, une cytolyse hépatique avec ASAT à $94 \mathrm{UI} / \mathrm{L}$ et ALAT $53 \mathrm{UI} / \mathrm{L}$, une cholestase très modérée avec un ictère (bilirubine totale $42 \mu \mathrm{mol} / \mathrm{L}$, bilirubine 
conjuguée $19 \mu \mathrm{mol} / \mathrm{L}$ ). Devant la bicytopénie observée, un frottis sanguin réalisé en hématologie révélait la présence d'éléments intra-érythrocytaires évocateurs de Plasmodium alors que l'amplification génique du genre Plasmodium (LAMP PCR) était négative.

À l'interrogatoire, aucune notion de voyage récent en zone impaludée n'a été retrouvée, remettant donc en question le diagnostic de paludisme. Les derniers voyages remontaient à 2004 au Japon, en Australie et en Inde sans notion d'antécédent de paludisme. Le diagnostic de babésiose est alors évoqué puis confirmé par l'analyse microbiologique du frottis sanguin coloré au May-Grünwald Giemsa et la PCR d'espèce en faveur de B. microti. La parasitémie était élevée à $7 \%$. On notait particulièrement des érythrocytes polyparasitées par des trophozoïtes piriformes, en tétrades ou vacuolés.

Un traitement par azithromycine $500 \mathrm{mg} / \mathrm{jour}$ et atovaquone $750 \mathrm{mg}$ matin et soir per os a été instauré avec une amélioration clinique et biologique rapide. Le patient était strictement apyrétique 24 heures après l'introduction du traitement. Le bilan biologique 48 heures après le début du traitement, montrait une parasitémie négative, une nette régression du syndrome inflammatoire (CRP $90 \mathrm{mg} / \mathrm{L}$ ) alors que l'anémie était plus marquée avec une hémoglobine à 6,8 $\mathrm{g} / \mathrm{dL}$. La babésiose est un diagnostic différentiel du paludisme qu'il faut évoquer chez tout patient présentant une fièvre inexpliquée sans notion de séjour en zone impaludée et ayant fréquenté dans les deux mois précédents un pays d'endémie durant la période d'activité des tiques au printemps ou à l'automne. D'un point de vue microbiologique, la présence d'un polyparasitisme des érythrocytes par des trophozoites piriformes, en tétrades ou vacuolés est fortement évocatrice de la babésiose. Le diagnostic microscopique doit être confirmé par PCR pour le diagnostic d'espèce.

\section{Session «Biologie hospitalière »}

\section{Deux années d'utilisation de la PCR grippe/VRS aux urgences : retour d'expérience}

Inès Rezzoug, Marlène Amara, Anne Gaëlle Cauchie, Amandine Hen, Stéphanie Marque Juillet

Service de biologie, Centre hospitalier André Mignot de Versailles, Le Chesnay, France

La grippe touche chaque année entre 2 et 8 millions de personnes en France. En période épidémique, les structures hospitalières ont besoin d'avoir un diagnostic rapide et fiable afin d'orienter les patients dans les lits d'avals et de désengorger le service des urgences. Ce diagnostic est particulièrement important dans les hôpitaux disposant majoritairement de chambres doubles. Ainsi, la délocalisation d'un automate de PCR grippe en période épidémique dans le service des urgences, après avoir mis en place des accords clinico-biologiques permettant de définir les conditions de réalisation du test, peut apporter une solution appropriée. L'objectif de notre étude est d'étudier l'impact de la mise en place d'un tel test.

\section{Matériel et méthode}

Etude rétrospective de deux saisons épidémiques sur l'appareil GeneXpert ${ }^{\circledR}$ (Cepheid) aux urgences.

Analyse de plusieurs paramètres :

- respect des indications définies dans les accords clinicobiologiques, à savoir : 1) PCR réalisée uniquement chez les patients dont la décision d'hospitalisation est déjà prise chez l'adulte ;2) PCR réalisée uniquement si le résultat de cette dernière modifie la prise en charge chez l'enfant ;

- délai moyen entre l'admission aux urgences et le rendu de la PCR en fonction de la période de réalisation du test. Nous avons étudié les dossiers de tous les patients ayant eu une PCR grippe aux urgences sur cette période.

\section{Résultats}

Au total, 2241 dossiers ont été étudiés. La mise en place de la PCR grippe en biologie délocalisée a permis de diminuer le délai de rendu de la PCR grippe avec un délai moyen de rendu de $6,3 \mathrm{~h}$ vs 4 à $72 \mathrm{~h}$ pour les prélèvements envoyés au laboratoire (virologie fermée la nuit, le WE, et les jours fériés) ; $42 \%$ des tests ont été réalisés en période de garde de week-end, parmi lesquels $69 \%$ ont été rendus dans un délai inférieur à $5 \mathrm{~h}$. Le délai de rendu des résultats en biologie délocalisée n'était pas influencé par le nombre de tests réalisés. Le nombre de prescriptions conformes aux accords clinico-biologiques chez l'adulte était de $84 \%$ la première année et de $83 \%$ la deuxième, sans différence entre les périodes ouvrables et les périodes de garde de week-end. En pédiatrie, le respect des indications était plus faible que chez l'adulte (58,6 et $65 \%)$.

\section{Conclusion}

La biologie délocalisée, nouvelle en microbiologie, présente un certain nombre de contraintes pour le biologiste (répondre à la norme 22870, formations des utilisateurs...). Néanmoins, les bénéfices apportés par la réalisation d'une PCR grippe au sein du service des urgences sont majeurs. En période d'épidémie de grippe, l'obtention d'un résultat rapide et fiable permet de faciliter la gestion des lits d'avals et donc de fluidifier les transferts et les hospitalisations de patients. Toutefois le contrôle et le suivi par le biologiste de ces tests coûteux sont indispensables pour éviter une dérive de leur utilisation. 


\section{Suivi du traitement de l'hémophilie $A$ : un dosage spécifique de l'activité du facteur VIII lonoctocog alfa faisant face aux limites des techniques de dosage traditionnelles}

\section{Floriane Devaux, Sara Zia-chahabi, Claire Flaujac}

Laboratoire d'hématologie biologique, Service de biologie médicale, Centre hospitalier de Versailles André-Mignot, Le Chesnay, France

L'Afstyla ${ }^{\circledR}$, lonoctocog alfa, est un facteur VIII (FVIII) recombinant humain de longue durée d'action utilisé dans le traitement de l'hémophilie A. Contrairement à d'autres produits, il n'y a pas d'ajout de macromolécules, il est constitué d'une chaîne unique avec un domaine B tronqué qui améliore sa stabilité. Pour son suivi, le fournisseur recommande une méthode chromogénique, difficilement applicable 24h/24, ou une méthode chronométrique en appliquant un facteur multiplicatif de 2, lié à une sousestimation de l'ordre de $45 \%$. L'utilisation d'une méthode chronométrique classique nécessite une comparaison préalable avec la méthode chromogénique et une vigilance lors de l'interprétation des résultats par les cliniciens devant appliquer le facteur multiplicatif. Cette étude a pour objectif d'évaluer la mise en place d'une méthode chronométrique, sans nécessité d'appliquer un facteur de conversion, grâce à une calibration spécifique du FVIII Afstyla®, permettant une disponibilité du dosage $24 \mathrm{~h} / 24$ avec nos réactifs habituels.

La calibration a été établie à partir d'Afstyla ${ }^{\circledR}$ reconstitué selon les recommandations fournisseur, puis dilué dans un plasma déficient en FVIII (concentration FVIII 1 UI/mL). Le plasma calibrant a été contrôlé par technique chronométrique, en tenant compte du facteur multiplicatif. La configuration du test (FVIII CKPA) sur l'automate Star Max® (Stago) est une courbe polynomiale d'ordre 2 , identique à celle habituellement utilisée. Les dosages de FVIII ont été réalisés par technique chronométrique (CK-Prest@, Stago (FVIII CKP)) ou par technique chromogénique TriniCHROM Factor VIII:C (Tcoag). La calibration FVIII CKPA obtenue est conforme à celle attendue avec le CKP. Les études de répétabilité et de reproductibilité ont été réalisées à partir de contrôles internes de qualité habituels : normal, pathologique, très pathologique et retrouve des coefficients de variation respectifs de répétabilité $(\mathrm{n}=10)$ de $1,24 \%, 1,32 \%$ et $2,96 \%$, et de reproductibilité $(\mathrm{n}=$ 12) de $6,2 \%, 4,0 \%$ et 7,7\% Ces résultats sont conformes aux critères acceptables du GFHT. La stabilité des réactifs à bord a été évaluée jusqu'à 5 h après reconstitution. Le FVIII Afstyla ${ }^{\circledR}$ reconstitué en eau est peu stable et passe de $120 \%$ à $103 \%$ en 1 h alors que le calibrant est stable jusqu'à 5 heures après reconstitution en plasma déficient FVIII. La linéarité a été vérifiée à partir de plasmas chargés en FVIII Afstyla ${ }^{\circledR}$ de $120 \%$ à $<1 \%(\mathrm{R} 2=1)$. La méthode a été vérifiée avec le plasma d'hémophile A sévère traité par Afstyla ${ }^{\circledR}$. Le FVIII par technique chromogénique $(80 \%)$ est comparable au FVIII CKPA (77\%), 2 fois plus élevée que le FVIII CKP (37 \%). La réalisation de la courbe de calibration dédiée Afstyla® est satisfaisante et peut constituer, dans la limite du coût du médicament utilisé pour la calibration, une alternative pour les laboratoires ne disposant pas de technique chromogénique et/ou souhaitant disposer d'un dosage $24 \mathrm{~h} / 24$ avec leurs réactifs de dosage chronométrique habituels.

\section{Validation d'une méthode de dosage simultané de l'imipénème, l'ertapénème et le méropénème dans le plasma humain par chromatographie liquide haute performance}

Amandine Caillault, Gaëlle Noé, Sybille Ghacham, Valérie Furlan

UF pharmacologie-toxicologie, Service de biochimiepharmacologie-toxicologie, Hôpital de Bicêtre, AP-HP, Le Kremlin-Bicêtre, France

Les carbapénèmes sont des bêta-lactamines à large spectre, de plus en plus utilisés en raison de l'émergence de bactéries résistantes, et utilisés dans le traitement probabiliste des infections sévères. La variabilité inter- et intra-individuelle de leurs paramètres pharmacocinétiques, conduisant à un risque de toxicité ou d'inefficacité, justifie leur suivi thérapeutique. La mise au point du dosage plasmatique des carbapénèmes permet de mesurer les concentrations résiduelles et maximales nécessaires à leur adaptation posologique. Une méthode de chromatographie liquide haute performance (CLHP) avec détection UV a été développée et validée selon les critères du Cofrac.

\section{Matériels et méthodes}

Le dosage est réalisé sur une chaîne CLHP Ultimate 3000, avec détection à $298 \mathrm{~nm}$, une colonne C18 et un gradient d'élution de tampon phosphate Na2HPO4 et de méthanol. Une gamme plasmatique $(0-100 \mathrm{mg} / \mathrm{L})$ stabilisée dans l'acide 3-morpholino-1-propanesulfonique (MOPS) et 3 contrôles de qualité (6-30-75 mg/L) ont été préparés. Le prétraitement consiste en une précipitation du plasma stabilisé contenant l'étalon interne (ceftazidime) avec de l'acétonitrile. Après centrifugation, on réalise une évaporation sous azote et une reprise dans le MOPS, $50 \mu \mathrm{L}$ sont injectés. La validation de méthode vérifie les critères suivants : rendement d'extraction, spécificité, linéarité, répétabilité, fidélité intermédiaire, exactitude, justesse, interférence avec d'autres médicaments $(n=68)$ et les substances endogènes, contaminations inter-échantillon, 
détermination de la limite de quantification (LOQ), vérification des différentes stabilités, et tests de comparaison inter-laboratoires.

\section{Résultats et discussion}

Les rendements d'extraction et les LOQ pour les 3 molécules sont respectivement $\geq 70 \%$ et $0,5 \mathrm{mg} / \mathrm{L}$, assurant une bonne sensibilité de la méthode pour les dosages en résiduel. La linéarité a été vérifiée jusque $100 \mathrm{mg} / \mathrm{L}$, permettant de doser aisément les concentrations maximales. La répétabilité, la justesse, la fidélité intermédiaire et l'exactitude sont correctes (coefficients de variation $\mathrm{CV}<9 \%$; biais $<7 \%$ ). Aucune interférence n'a été démontrée permettant le dosage chez des patients souvent polymédicamentés. Les conditions chromatographiques assurent une bonne sélectivité des 4 pics sur un run de 30 minutes, restant une limite de la méthode. Les stabilités diffèrent entre les carbapénèmes excepté celle des 3 congélations/décongélations à $-80{ }^{\circ} \mathrm{C}$ permettant de redoser des échantillons si nécessaire. L'imipénème n'est stable que $24 \mathrm{~h}$ à $4^{\circ} \mathrm{C}$, les 2 autres carbapénèmes sont stables 4 jours à $4{ }^{\circ} \mathrm{C}$ et $24 \mathrm{~h}$ à $20^{\circ} \mathrm{C}$.

\section{Conclusion}

Cette méthode de dosage simultané de l'imipénème, ertapénème et méropénème en milieu plasmatique est simple, exacte, reproductible et spécifique. Elle constitue un outil important du suivi thérapeutique pharmacologique chez les patients atteints d'infections compliquées.

\section{Mise au point au laboratoire du diagnostic de l'ataxie cérébelleuse autosomique dominante (ADCA) SCA36 : le gène NOP56 arrive au $5^{e}$ rang des gènes les plus fréquemment impliqués dans les ADCA}

Marie Temple*, Jean-Madeleine De Saint-Agathe, Kathy Larcher, Sandrine Noel, Eric Le Guern, Cécile Cazeneuve

*Lauréat du Prix AAIIPHP

Département de génétique, UF de neurogénétique, GH Sorbonne Université, Hôpital Pitié-Salpêtrière, AP-HP, Paris, France

Les ataxies cérébelleuses autosomiques dominantes (ADCA) constituent un ensemble hétérogène tant d'un point de vue clinique que génétique.

Les gènes ATXN1, ATXN2, ATXN3, CACNA1A, ATXN7, TBP et ATN1 sont les plus fréquemment impliqués. Cependant, au laboratoire de diagnostic, seuls environ $32 \%$ des patients adressés pour une forme familiale d'ADCA portent une mutation (expansion de triplets CAG) dans un de ces gènes testés. Le gène NOP56 a été rapporté comme étant le gène causal de l'ADCA SCA36. La mutation en cause est une grande expansion d'hexanucléotides GGCCTG dans l'intron 1 du gène.

Nous avons mis au point le diagnostic de SCA36 au laboratoire avec deux techniques complémentaires : 1) une repeat-primed PCR (RP-PCR) pour la mise en évidence des grandes expansions et la limite de détection de la RP-PCR n'étant pas connue, 2) une PCR quantitative permettant de doser le nombre d'allèles de taille normale. Ces techniques ont été appliquées à une grande cohorte d'environ 400 patients adressés pour une forme familiale d'ADCA, pour lesquels la recherche au laboratoire de mutation des 7 gènes les plus fréquemment impliqués dans les ADCA s'était révélée négative.

Les résultats préliminaires portant sur environ $95 \%$ des familles d'intérêt montrent qu'une grande expansion d'hexanucléotides du gène NOP56 est retrouvée dans 20 familles soit un nouveau diagnostic pour 32 patients. Cette fréquence place le gène NOP56 au 5e rang des gènes les plus fréquemment impliqués dans les ADCA dans la population testée, après les gènes ATXN3, ATXN2, ATXN1 et ATXN7, respectivement au 1er, $2 \mathrm{e}, 3 \mathrm{e}$ et $4 \mathrm{e}$ rang avec 101, 62,37 et 25 familles. Aucun signe clinique n'est spécifique de SCA36 dans les 20 familles identifiées ; cependant, une hypoacousie et, à un moindre degré, des fasciculations de la langue ou de la bouche semblent être plus fréquemment associées à SCA36 qu'aux autres SCA.

De façon surprenante, la présence d'une petite expansion associée en mosaïque à une grande expansion (GE) (nombre de répétitions $>650$ répétitions) a été observée chez 3 patients indépendants. Dans l'une de ces familles, sont observés un patient porteur d'une petite expansion sans mosaïque (31 répétitions), une patiente, nièce du précédent, porteuse d'un allèle avec 23 répétitions associé en mosaïque avec une GE et plusieurs patients (dont deux sont de la même fratrie que la patiente précédente) porteur d'une GE. Ces observations apportent des éléments nouveaux dans la physiopathologie moléculaire de SCA36.

Compte tenu de la fréquence de la mutation du gène NOP56 dans ces familles atteintes d'ADCA, nous proposons d'inclure l'étude de ce gène dans la stratégie diagnostique des ADCA effectuée en routine au laboratoire.

\section{Mise au point et applications diagnostiques d'une technique de flux métabolique de la bêta-oxydation mitochondriale des acides gras sur sang total}

Edouard Le Guillou ${ }^{l}$, Dimitri Schlemmer ${ }^{l}$, Jean-François Benoist $^{1,2}$, Apolline Imbard ${ }^{1,2}$

1 Laboratoire de biochimie-hormonologie, Centre hospitalo-universitaire Robert Debré, AP-HP, Paris, 
France ; 2 Lip(Sys)2, Faculté de pharmacie, Université Paris-Saclay, Châtenay-Malabry, France

Les maladies héréditaires de la bêta-oxydation des acides gras résultent du déficit d'une enzyme ou d'un transporteur de cette voie métabolique. Le diagnostic repose sur l'association de signes cliniques (troubles hépatiques, myopathies) et biochimiques (hypoglycémie, anomalies du profil des acylcarnitines) et est confirmé par biologie moléculaire. Dans certains cas, le diagnostic reste difficile avec des présentations cliniques peu spécifiques, des profils biochimiques non informatifs car prélevés en dehors des épisodes aigus ou complexes à interpréter, et des variants génétiques de signification indéterminée. Des études fonctionnelles mesurant le flux intracellulaire de la bêta-oxydation sont apparues récemment. A partir d'une méthode développée par Dessein et al., nous avons mis au point une technique rapide de mesure du flux métabolique de la bêta-oxydation, par quantification des acylcarnitines marquées produites dans les cellules sanguines des patients après incubation avec du palmitate marqué avec des isotopes stables.

\section{Matériels et méthodes}

Cent $\mu \mathrm{L}$ de sang total sont incubés pendant $6 \mathrm{~h}$ à $37{ }^{\circ} \mathrm{C}$ en présence de $200 \mathrm{nmol}$ de d9-palmitate et $500 \mathrm{nmol}$ de L-carnitine. Les réactions sont stoppées par congélation immédiate, les échantillons sont ensuite déprotéinisés, soniqués et centrifugés. Les acylcarnitines deutérées produites (de $\mathrm{C} 2$ à C16) sont dosées dans le surnageant par chromatographie liquide couplée à la spectrométrie de masse en tandem (LC-MS/MS), et quantifiées par dilution isotopique. Les résultats sont exprimés en nmol/G leucocytes et en rapport au témoin (\%). Nous avons établi des valeurs de référence pour chaque acylcarnitine à partir de 43 sujets sains. Des patients avec déficits connus ont été testés : navette de la carnitine (CPT-II, $\mathrm{n}=1$ ), chaîne longue (VLCAD, $\mathrm{n}=2$ ), chaîne moyenne (MCAD, $\mathrm{n}=2$ ).

\section{Résultats}

Nous avons mis au point une technique LC-MS/MS et optimisé les conditions analytiques : temps d'incubation, extraction des acylcarnitines, concentration en substrat, choix d'étalons internes, durée de conservation pré-incubation. Cette technique permet de quantifier spécifiquement chaque acylcarnitine. Des rapports entre acylcarnitines permettent de sensibiliser le diagnostic. Nous retrouvons une augmentation de $8100 \%$ par rapport à la médiane des témoins du rapport C16/(Somme C2 à C14) en cas de déficit en CPT-II/translocase, de $2600 \%$ du rapport C14/(Somme C2 à C12) en cas de déficit en VLCAD et de $226200 \%$ du rapport C8/C4 en cas de déficit en MCAD.

\section{Conclusion}

Nous avons développé une technique de mesure in vitro du flux de la bêta-oxydation permettant de discriminer des patients atteints de déficit de la bêta-oxydation de sujets sains. Celle-ci a été mise en place en routine et a déjà permis de conclure sur certains cas difficiles. Cette technique constitue par ailleurs un outil important pour la validation fonctionnelle des variants identifiés par biologie moléculaire.

\section{Session « Recherche clinique, fondamentale et translationnelle »}

\section{Caractérisation in vitro et in vivo d'un anticorps anti-thrombine responsable d'un syndrome hémorragique sévère chez un patient atteint de gammapathie monoclonale d'origine indéterminée}

Bérénice Schell, Thomas Brungs, Abla Amara, Céline Desconclois, Cécile Lavenu Bombled, Cécile Goujard, Xavier Mariette, Valérie Proulle

Laboratoire d'hématologie biologique, CHU Paris-Sud Hôpital de Bicêtre, Le Kremlin Bicêtre, France

Les anticorps anti-thrombine (ATA) sont une cause rare de troubles acquis de la coagulation. Nous présentons la caractérisation in vitro et in vivo d'un ATA responsable d'un syndrome hémorragique sévère chez un patient atteint de gammapathie monoclonale d'origine indéterminée de type $\operatorname{IgG} \kappa$.

In vitro, l'activité anti-thrombine (anti-IIa) du plasma pauvre en plaquettes (PPP) a été identifiée par les temps de céphaline activée (TCA) et temps de thrombine (TT). Le test de génération de thrombine (TGT) sur PPP a été effectué, mesurant le temps de latence (LT) et le potentiel de thrombine endogène (ETP), sur l'appareil CAT®, Stago. Les IgG anti-IIa ont été purifiées en chromatographie d'affinité par fixation sur $\alpha$ IIa humaine. Leur effet in vivo a été étudié sur la formation du thrombus dans un modèle murin de thrombose artérielle induite par laser : l'accumulation plaquettaire et la fibrino-formation au site de lésion ont été étudiées en microscopie intra-vitale avec des anticorps anti-plaquettes et anti-fibrine conjugués à des fluorochromes. In vitro le TCA et le TT du patient sont allongés à $76 \mathrm{~s}$ et $>120 \mathrm{~s}$ (contrôles $35 \mathrm{~s}$ et $18 \mathrm{~s}$ ). Les résultats du dosage des facteurs de la coagulation sont normaux, aucun traitement anticoagulant n'est détecté. Le TT n'est pas corrigé par l'addition de PPP témoin, suggérant la présence d'un ATA. Le TGT effectué avec le PPP patient reste plat durant $1 \mathrm{~h}$ (LT > $1 \mathrm{~h}, \mathrm{ETP}=0$ ). Le TGT d'un mélange de dilutions croissantes 
(1:1 à 1:32) de PPP patient avec un PPP contrôle objective un raccourcissement du LT (de 38 à $6 \mathrm{~min}$ ) et une augmentation de l'ETP (251 à $575 \mathrm{nM} / \mathrm{min}$ ) sans atteindre les valeurs obtenues avec le PPP témoin (4 min et $1183 \mathrm{nM} / \mathrm{min}$ ). Ceci confirme l'activité anti-coagulante du PPP du patient.

Comparé à l'ajout d'IgG contrôles dans un PPP témoin, l'ajout d'IgG anti-IIa purifiées à partir du plasma du patient entraîne un allongement du TCA à 59 s (vs 33 s) et du TT $>120 \mathrm{~s}$ (vs $19 \mathrm{~s}$ ). Ces résultats montrent la responsabilité des IgG anti-IIa dans les anomalies plasmatiques. In vivo, dans le modèle murin de thrombose, comparée à l'injection intraveineuse d' IgG contrôles (1 mg), l'injection intraveineuse d'IgG anti-IIa purifiées (690 $\mu \mathrm{g}$ ) entraîne une diminution d'au moins 70 \% de l'accumulation plaquettaire et de la fibrinoformation au site de la lésion vasculaire, illustrant l'effet inhibiteur observé in vitro.

En conclusion, chez un patient présentant un syndrome hémorragique et une $\operatorname{IgG} \kappa$ monoclonale, nous avons mis en évidence in vitro une activité anti-IIa du plasma sur les tests spécifiques et globaux de l'hémostase. Nous avons montré que les IgG anti-IIa purifiées à partir du plasma du patient provoquaient, in vitro, un effet anti-coagulant et, in vivo, une inhibition de la formation du thrombus dans un modèle murin de thrombose. Ces résultats illustrent de manière unique l'imputabilité d'un ATA dans l'apparition d'un syndrome hémorragique chez un patient et la corrélation entre la clinique et la biologie, in vitro, ex vivo et in vivo.

\section{Recherche de gènes modificateurs dans la neurofibromatose de type 1 par étude d'association génome entier}

Laurence Pacot ${ }^{1,2}$ *, Audrey Sabbagh ${ }^{3 *}$, Béatrice Parfait ${ }^{1,2}$, Anne Boland-Auge ${ }^{4}$, Delphine Bacq-Daian, ${ }^{4}$ Ingrid Laurendeau ${ }^{2}$, Salah Ferkal ${ }^{5,6}$, Audrey Briand ${ }^{1,2}$, Laurence Allanore ${ }^{5}$, Jean-François Deleuze ${ }^{4}$, Michel Vidaud $^{1,2}$, Dominique Vidaud ${ }^{1,2}$, Réseau Nf-France, Pierre Wolkenstein $^{5,6,7}$, Éric Pasmant ${ }^{1,2}$

*Contribution égale des auteurs

1 Service de génétique et biologie moléculaires, Hôpital Cochin, AP-HP, Centre Université de Paris, Paris, France ; 2 Inserm UMR_S1016, Institut Cochin, Université de Paris, Paris, France ; 3 UMR 261 MERIT, Institut de recherche pour le développement, Université de Paris, Paris, France ; 4 CEA, Centre national de recherche en génomique humaine, Evry, France ; 5 Département de dermatologie, Centre de référence des neurofibromatoses, Hôpital HenriMondor, AP-HP, Créteil, France ; 6 CIC1430, Hôpital
Henri-Mondor, AP-HP, Créteil, France ; 7 EA7379, Université Paris-Est-Créteil, Créteil, France

La neurofibromatose de type 1 (NF1) est une maladie génétique caractérisée par le développement de tumeurs bénignes des gaines nerveuses appelés neurofibromes, qui peuvent se transformer en cancers appelés MPNST (malignant peripheral nerve sheath tumors). Ces MPNST sont la première cause de mortalité chez les patients atteints de NF1. Transmise sur un mode autosomique dominant, la NF1 est causée par la perte de fonction du gène suppresseur de tumeur NF1 dans les cellules de Schwann. Ce gène code la neurofibromine, régulateur négatif de la voie RAS-MAPK. Malgré une pénétrance complète à l'âge de 8 ans, l'expressivité de la maladie est extrêmement variable, y compris au sein d'une même famille. De rares variants faux-sens du gène NF1 ont pu être corrélés à des formes plus ou moins sévères de NF1, mais la plus grande part de la variabilité phénotypique de cette maladie reste inexpliquée à ce jour. L'étude de modèles animaux et de corrélations phénotypiques intrafamiliales a suggéré l'existence de gènes modificateurs dans la NF1. Nous présentons ici les résultats de la première étude d'association génome entier pour la NF1, réalisée en collaboration avec le réseau NF-France. Ce travail a reposé sur l'étude génotypique et phénotypique de plus de 1500 patients atteints de NF1 issus de 3 programmes hospitaliers de recherche clinique entre 2003 et 2013. Seuls les patients porteurs de variants pathogènes du gène NF1 pour lesquels aucune corrélation n'a été précédemment établie ont été retenus et génotypés. Après un contrôle de qualité des données, une cohorte de 1333 patients a été obtenue et subdivisée en 2 échantillons de 918 (échantillon de découverte, ED) et 415 patients (échantillon de réplication, ER). Notre étude s'est focalisée sur 3 phénotypes majeurs dans la NF1 : les neurofibromes cutanés, souscutanés et plexiformes. L'analyse des données montre que le seuil de signification statistique à l'échelle du génome ( $p<5.10-8$ ) est franchi dans l'ED pour le trait des neurofibromes plexiformes. Par ailleurs, bien qu' inférieurs au seuil pangénomique, d'autres signaux significatifs sont observés dans les différents phénotypes étudiés, avec un total de 10 régions génomiques répliquées $(\mathrm{p}<10-5)$ et de 22 régions significatives non répliquées $(\mathrm{p}<10-6)$ observées pour l'ensemble des 3 traits étudiés. Ces régions pointent l'implication potentielle respectivement de 83 et 270 gènes, dont 40 et 116 gènes codants. Parmi eux, plusieurs sont impliqués ou en lien avec la voie RAS-MAPK (WDR48, MAP3K7, MMD2, TBK1, RAP2A), le cycle cellulaire (DAPK1, GAS1, FYN, MYCL) ou le processus de myélinisation (FRMD4A, FYN).

Notre étude permet de confirmer l'implication de gènes précédemment identifiés et révèle plusieurs gènes modi- 
ficateurs potentiels dans la NF1. Des régions génomiques à risque distinctes entre les trois phénotypes étudiés sont mises en évidence, suggérant l'existence de mécanismes physiopathologiques spécifiques à l'origine de chaque type de neurofibromes.

\section{Intérêt de l'évaluation de la réponse immunitaire spécifique anti-cytomégalovirus (CMV) par le test QuantiFERON®-CMV dans la prise en charge des patients transplantés rénaux}

Clémentine Moulin*, Claire Deback, Séverine Beaudreuil, Hugo Roux, Salima Hacein-Bey-Abina, Aude Gleizes

* Lauréat du Prix du SIPHIF

Laboratoire d'immunologie, CHU Paris-Sud - Hôpital de Bicêtre, AP-HP, Le Kremlin-Bicêtre, France

L'infection à CMV, complication infectieuse la plus fréquente du traitement immunosuppresseur des receveurs d'organes, est responsable d'une forte morbidité et d'une augmentation du risque de rejet. Les traitements antiviraux ont des effets indésirables conduisant parfois à leur arrêt prématuré ou à l'émergence de résistances compliquant la prise en charge. L'immunité cellulaire médiée par les lymphocytes T CD8 (LT CD8) constitue le mécanisme majeur de défense anti-CMV. Le test QuantiFERON®CMV (QTF-CMV) est une méthode Elisa qui permet l'analyse de la réponse immunitaire anti-CMV en mesurant la sécrétion d'IFN $\gamma$ des LT CD8 effecteurs mémoires après leur stimulation par des peptides viraux.

L'objectif de ce travail est d'évaluer l'apport du QTF-CMV pour détecter la présence d'une immunité $\mathrm{T}$ spécifique protectrice afin d'optimiser la prise en charge thérapeutique des patients transplantés rénaux. Au total, 63 patients greffés rénaux ont été inclus constituant 3 groupes de risque CMV définis selon le statut sérologique CMV : 6 D-/R- (risque faible) ; $22 \mathrm{D}-/ \mathrm{R}+$ et $17 \mathrm{D}+/ \mathrm{R}+$ (risque modéré) ; $18 \mathrm{D}+/ \mathrm{R}$ (haut risque). Le QTF-CMV a été réalisé au cours du suivi post-greffe et pour 25 d'entre eux avant transplantation (J0). Les tests sont réalisés et interprétés selon les recommandations du fabricant : négatif (pas d'immunité anti-CMV), positif (immunité anti-CMV), indéterminé (non interprétable). Les charges virales (CV), renseignements cliniques et traitements antiviraux ont été colligés.

Nous montrons qu'il existe une bonne corrélation entre le QTF-CMV et le statut sérologique puisqu'à J0, tous les patients R- ont un QTF-CMV négatif et sur les 15 patients R+, 12 ont un QTF-CMV positif, soit $88 \%$ de concordance. Trois patients $\mathrm{R}+$ ont par contre une immunité cellulaire anti-CMV altérée (QTF-CMV négatif), ce qui est cohérent avec la littérature et témoigne d'un risque majoré d'infection post-greffe.

Parmi les 7 patients ayant eu une infection à CMV, 2 avaient un QTF-CMV indéterminé, 3 négatif et 2 positif. Le risque infectieux semble donc plus fréquent en l'absence d'immunité anti-CMV. L'intérêt du monitoring par le QTFCMV sur la stratégie de prise en charge est mis en évidence par 2 exemples : un patient $\mathrm{D}+/ \mathrm{R}$ - sous prophylaxie CMV, présentant un QTF-CMV négatif à un mois de la greffe et une $\mathrm{CV}$ indétectable, chez lequel le virus réplique 4 mois plus tard. La réponse anti-CMV testée plusieurs fois négative ne permet pas de contrôler l'infection malgré plusieurs lignes de traitement anti-CMV. A contrario, un patient $\mathrm{D}+/ \mathrm{R}+$ ayant une forte réponse $\mathrm{T}$ anti-CMV à 11 mois de la greffe conserve un contrôle de la réplication virale malgré l'arrêt de la prophylaxie depuis 4 mois.

En conclusion, le QTF-CMV permet de compléter les données du statut sérologique pour une meilleure stratification des patients à risque. De plus, ce test pourrait aider en posttransplantation à optimiser la prise en charge thérapeutique en améliorant l'évaluation du risque de réplication virale.

\section{Dysfonctions mitochondriales dans les macrophages spumeux induites par la surcharge en LDL-cholestérol}

Edouard Le Guillou ${ }^{1,2} *$, Pierre-Hadrien Becker ${ }^{1,2}$, Patrice Thérond $^{1,2}$, Pauline Gaignard ${ }^{1,2}$

*Lauréat du Prix Adebiopharm

1 Unité universitaire interdisciplinaire Lip (Sys) ${ }^{2}$, Université Paris-Saclay, Châtenay-Malabry, France ; 2 Service de biochimie, CHU Bicêtre, AP-HP, Université Paris-Saclay, Le Kremlin-Bicêtre, France

Le métabolisme mitochondrial constitue une nouvelle voie de recherche prometteuse dans la physiopathologie de l'athérosclérose : en effet, il a été montré dans certains modèles cellulaires que la surcharge en cholestérol altère le fonctionnement de la chaîne respiratoire mitochondriale (CRM), principale source d'ATP ; or, l'efflux du cholestérol des macrophages spumeux est majoritairement ATP-dépendant. Notre hypothèse est donc que la surcharge en LDL-cholestérol altèrerait le fonctionnement mitochondrial des macrophages.

\section{Matériels et méthodes}

Des macrophages humains THP-1 ont été surchargés ou non par des LDL acétylées (LDLac, modèle de lésion précoce d'athérosclérose) ou oxydées (LDLox, modèle de lésion tardive). Les concentrations de glucose et lactate dans le milieu de culture ont été mesurées après $48 \mathrm{~h}$ de surcharge. 
Une analyse en cinétique du stress oxydant mitochondrial a été réalisée par mesure du glutathion cellulaire et mitochondrial par spectrométrie de masse et de la production d'anions superoxydes par fluorimétrie à $4 \mathrm{~h}, 24 \mathrm{~h}$ et $48 \mathrm{~h}$ de surcharge. L'activité de l'aconitase, une enzyme du cycle de Krebs inactivée par le stress oxydant, a été mesurée à $48 \mathrm{~h}$ par spectrophotométrie ( $\mathrm{n}=6$ séries). Un test Anova avec post-test de Tukey a été utilisé pour l'analyse statistique.

\section{Résultats}

Des travaux antérieurs de notre équipe ont montré que la surcharge pendant $48 \mathrm{~h}$ en LDLac et LDLox diminue la consommation d'oxygène par la CRM par rapport aux cellules témoins et que cette diminution est plus marquée en présence de LDLox qu'en présence de LDLac. L'augmentation de la quantité de lactate produit par quantité de glucose consommée indique que le métabolisme majoritaire est de type glycolytique après surcharge en LDLox (augmentation de $49 \%$ du ratio lactate produit/glucose consommé, $\mathrm{p}<0,001$ vs non surchargé). La production d'anions superoxydes n'est pas modifiée significativement par la surcharge au cours du temps. Cependant, l'augmentation de glutathion réduit dans la cellule dès $4 \mathrm{~h}$ $(+37 \%$ en présence de LDLac, $\mathrm{p}<0,05)$ et à $24 \mathrm{~h}(+135 \%$ LDLac $\mathrm{p}<0,01 ;+161 \%$ LDLox, $\mathrm{p}<0,001$ ), ainsi que l'accumulation de glutathion oxydé dans la mitochondrie en présence de LDLox $(+440 \%$ à $24 \mathrm{~h}, \mathrm{p}<0,01 ;+470$ $\%$ à $48 \mathrm{~h}, \mathrm{p}<0,001)$ traduisent un état de stress oxydant, plus important en présence de LDLox que de LDLac. Il est observé en parallèle une diminution de l'activité de l'aconitase à 48h (- 26\% LDLac, p < 0,001; - $72 \%$ LDLox, $\mathrm{p}<0,001)$, très probablement par dommages oxydatifs.

\section{Conclusion}

Ces résultats montrent que la surcharge en LDL dans les macrophages induit un stress oxydant mitochondrial concomitant à la diminution de la consommation d'oxygène par la CRM. Ces dysfonctions mitochondriales sont plus marquées en présence de LDLox, modèle physiopathologique qui correspond à un état d'athérosclérose avancée. Ce travail se poursuit par l'analyse des effets sur la CRM des oxystérols, produits d'oxydation du cholestérol.

\section{La réversion génétique dans la maladie de Fanconi, un modèle naturel de thérapie génique}

Lise Larcher ${ }^{1}$, Anna Raimbault ${ }^{2}$, Nadia Vasquez ${ }^{2}$, Mélanie Da Costa ${ }^{2}$, Samuel Quentin ${ }^{3}$, Lucie Hernandez $z^{3}$, Catherine Dubois d'Enghien ${ }^{4}$, Marie Sébert ${ }^{3}$, Thierry Leblanc ${ }^{5}$, Dominique Stoppa-Lyonnet ${ }^{6}$, Régis Peffault de Latour ${ }^{7}$, Jean Soulier ${ }^{3}$
1 Inserm/CNRS U944/7212, Institut de recherche SaintLouis, Université de Paris, France ; 2 Laboratoire d'hématologie, Hôpital Saint-Louis, AP-HP, Paris, France ; 3 Inserm/CNRS U944/7212, Institut de recherche SaintLouis, Université de Paris \& Laboratoire d'hématologie, Hôpital Saint-Louis, AP-HP, Paris, France ; 4 Service de génétique, Institut Curie Hôpital, Paris, France ; 5 Hematology Unit, Hôpital Robert Debré, AP-HP, Paris, France ; 6 DRUM Team, Inserm U830, Institut Curie, Paris, France \& Service de génétique, Institut Curie Hôpital, Paris, France \& Université de Paris, France ; 7 Centre de référence aplasies médullaires \& Service d'hématologiegreffe de moelle, Hôpital Saint-Louis, AP-HP, Paris, France

La maladie de Fanconi ( $\mathrm{AF}$ ) est une maladie génétique récessive caractérisée par une fragilité chromosomique, l'apparition progressive d'une aplasie médullaire et une prédisposition aux cancers. A ce jour, 22 gènes FANC ont été impliqués dans l'AF. Typiquement, les patients AF développent une aplasie médullaire à un âge médian de 7 ans dont le seul traitement curatif était jusqu'à présent la greffe de cellules souches hématopoḯtiques (HSCT). Or l'HSCT est un traitement lourd aux effets indésirables graves et fréquents. Pour la première fois, des essais cliniques de thérapie génique (GT) ont montré des résultats précoces encourageants chez des patients AF (Río et al., Nat Med 2019). Cependant, les patients AF ont un risque intrinsèque élevé de développer une leucémie aiguë myéloïde (LAM). Quid de ce risque chez les patients ayant une fraction de cellules hématopoïétiques génétiquement corrigées par GT et qui ne seront pas greffés ? Pour évaluer ce risque à moyen et long terme, nous avons étudié une situation naturelle qui survient spontanément dans une faible proportion de patients $\mathrm{AF}$ : la réversion somatique, ou mosaïcisme somatique hématopoïétique, due à l'acquisition d'un événement génétique conduisant à l'émergence de cellules spontanément corrigées. Cet événement rétablit la fonctionnalité du gène FANC et confère aux cellules porteuses une résistance aux agents génotoxiques et un avantage sélectif.

Nous avons sélectionné 13 patients, au sein d'une cohorte nationale de 335 patients $\mathrm{AF}$, qui présentaient une normalisation d'un test fonctionnel au niveau sanguin. Nous avons re-séquencé par NGS un panel de 62 gènes (FANCA, le gène le plus fréquemment muté dans l'AF + 61 gènes associés aux hémopathies myéloïdes) dans l'ADN extrait de cellules sanguines et comparé les données à celles du matériel constitutionnel (fibroblastes cutanés). Pour 12/13 patients, nous avons mis en évidence de façon certaine la réversion génétique qui se produisait le plus fréquemment par conversion génique. L'analyse des données cliniques montre qu'aucun des 13 patients n'a développé de LAM à 


\section{Congrès}

un âge médian de 25 ans [12-61]. Néanmoins, 4 (31\%) ont développé un cancer solide à un âge médian de 39 ans.

Ainsi, au sein de notre cohorte de patients révertants fonctionnels, nous avons pu identifier par une capture NGS la réversion génétique de FANCA, et montré la meilleure évolution hématologique de ces patients. De façon importante, le mosaïcisme hématopoiétique semble protéger de l'apparition de LAM mais pas des cancers solides. Les données de suivi à moyen et long terme de ces patients révertants encouragent la mise en œuvre de la GT dans l'AF. 